Pacific Journal of Mathematics

CONCRETE SEMISPACES AND LEXICOGRAPHIC
SEPARATION OF CONVEX SETS 


\title{
CONCRETE SEMISPACES AND LEXICOGRAPHIC SEPARATION OF CONVEX SETS
}

\author{
C. EDWARD MOORE
}

\begin{abstract}
V. L. Klee raised a question concerning lexicographic separation of disjoint closed convex sets in a locally convex space by means of a semispace with a representation utilizing continuous linear functionals. This question is answered and related results involving hyperplane separation and reflexivity in Banach spaces are discussed.
\end{abstract}

1. Introduction. If $p$ is a vector in a real linear space $E$, then a semispace at $p$ is a maximal convex subset of $E \sim\{p\}$. This notion was introduced by Hammer [3] in 1955 and the structure of semispaces was determined by Klee [7] in 1956. The present paper is strongly dependent upon this work of Klee. The concept of a concrete semispace in a locally convex space is introduced and a basic representation theorem for such an object is proved. Concrete separation (lexicographic separation via concrete semispaces) is defined and a criterion is given for determining when two sets are not concretely separated. Disjoint closed convex subsets $A$ and $B$ of a pre-Hilbert space $E$ such that $A-B$ is dense in $E$ and $A-B$ has nonempty core are exhibited. These sets cannot be separated concretely and this implies a negative answer to Klee's question concerning concrete separation of disjoint closed convex sets in a locally convex space. Hyperplane separation and concrete separation are contrasted. It is proved that a Banach space $E$ is reflexive if and only if each disjoint pair of bounded closed convex subsets of $E$ is concretely separated.

2. Preliminaries. Only real linear spaces will be considered and these will be denoted by $E$. The real numbers will be denoted by $\boldsymbol{R}$ and the natural numbers by $\boldsymbol{N}$. We write the linear span of a subset $X$ of $E$ as $\operatorname{sp}(X)$. If $S$ is a semispace at $p$ in $E$ then $S$ is a maximal convex cone with vertex $p$ (deleted) and hence Köthe [9] calls these objects hypercones. The reflection $S^{*}=2 p-S$ of $S$ is also a semispace at $p$ and $S^{*}$ is situated so that $S^{*} \cap S=\varnothing$ and $E \sim\{p\}=S \cup S^{*}$. When studying the structure of semispaces it is sufficient to consider the case where $p$ is the zero vector $\theta$ since $S$ is a semispace at $p$ if and only if $-p+S$ is a semispace at $\theta$. Hereafter, unless mention is made to the contrary, "semispace" will mean semispace at $\theta$.

If $A$ is a nontrivial subset of $E$, write $x \rightarrow y$ for $x, y \in A$ if there exists $z \in A \sim\{y\}$ such that the closed line segment $[x, y]$ is contained 
in $[x, z]$ and $[x, z] \subset A$. In this case we say that $x$ can see past $y$ in $A$. Write $x \rightarrow \rightarrow^{\prime} y$ provided $x \rightarrow y$ but not $y \rightarrow x$. If $A$ is convex and $x \approx y$ is written whenever $x \rightarrow y$ and $y \rightarrow x$ then $\approx$ is an equivalence relation on $A$. If $S$ is a semispace in $E$ denote by $\Gamma$ the equivalence classes of $S$ determined by $\approx$ and observe that, when transferred to $\Gamma, \rightarrow$ becomes a reflexive partial order. Each member of $\Gamma$ is a convex set.

A vector $x$ in a set $A$ is a core point of $A$ if each line in $E$ through $x$ contains an open line segment about $x$ which lies in $A$. The set of all core points of $A$ is denoted by core $(A)$. If $A$ is convex and $x \in \operatorname{core}(A)$ and $y \in A$, then the interval $[x, y)=\{t x+(1-t) y: 0<$ $t \leqq 1\}$ lies in core $(A)$. Applying these ideas to a semispace $S$, we see that if core $(S) \neq \varnothing$, then core $(S) \in \Gamma$ and core $(S)$ is the maximal [minimal] element of $\Gamma$ with respect to $\rightarrow[-]$. The maximality is due to the fact that if $y \notin \operatorname{core}(S)$, then no member $x$ of core $(S)$ can see past $y$ in $S$.

We list Theorems 2.1-2.4 which were established by Klee [7]. No originality is claimed by the present author for any of this material, but it is included here in order to provide the necessary basis for later definitions and results.

TheOREM [Klee] 2.1. If $M$ is a linear subspace of $E$ and $T \subset M$, then $T$ is a semispace in $M$ if and only if $T=S \cap M$ for some semispace $S$ in $E$. If $H$ is a hyperplane in $E, Q$ is one of the open halfspaces determined by $H$ and $T$ is a semispace in $H$, then $T \cup Q$ is a semispace in $E$. Conversely, if $E$ is finite-dimensional then every semispace in $E$ has this form.

THEOREM [Klee] 2.2. Suppose $S$ is a semispace in $E$ and $\eta \in \Gamma$. If $\xi \in \Gamma$, write $\xi \rightarrow^{\prime} \eta$ provided $x \rightarrow^{\prime} y$ whenever $x \in \xi$ and $y \in \eta$ and let $A$ denote the union of all $\xi \rightarrow \rightarrow^{\prime} \eta$. If $E_{\eta}=\mathrm{sp}(\eta)$ then:

(a) $\eta$ is an open halfspace in $E_{\eta}$ whose bounding hyperplane contains $A$, so that $\eta=$ core $(\eta)$ in $E_{\eta}$;

(b) $A$ is convex;

(c) $S \cap E_{\eta}=A \cup \eta$.

An anti-reflexive linear order $r$ for a total collection $F$ of linear functionals on $E$ is said to be usable for $F$ if for each nonzero vector $x$ in $E$ there exists a first member $f_{x}$ in $F$ such that $f_{x}(x) \neq 0$. In this case we define

$$
S(F, r)=\left\{x \in E: f_{x}(x)>0\right\}
$$

and note that $S(F, r)$ is a semispace at the origin $\theta$ in $E$. 
KLEE REPRESENTATION THEOREM 2.3. If $S$ is a semispace in $E$, there exist a set $F$ of linear functionals on $E$ and a linear order $r$ on $F$ such that $S=S(F, r)$.

In Klee's proof of 2.3 each $f \in F$ is a first functional $f_{x}$ for some nonzero $x$ in $E$. A representation $S(F, r)$ for $S$ with this property is called a Klee representation. By deleting the unused functionals from $G$ in an arbitrary representation $S\left(G, r^{\prime}\right)$ and restricting the order $r^{\prime}$, we obtain a Klee representation for $S$.

TheOREM [Klee] 2.4. Suppose $S(F, r)$ is a Klee representation for a semispace $S$ in $E$. If $x, y \in S$ then

(a) $x \rightarrow ' y$ if and only if $f_{y} r f_{x}$;

(b) $x \approx y$ if and only if $f_{x}=f_{y}$.

Thus, if in $2.4 x \in \eta \in \Gamma$, then $f_{x}=f_{y}$ for all $y \in \eta$ and there is a one-to-one correspondence between members of $\Gamma$ and elements of $F$ when $S(F, r)$ is a Klee representation for $S$. Now suppose core $(S) \neq$ $\varnothing$ and note that $F$ must have a first functional $f_{1}$ since the linear order $r$ on $F$ satisfies

$$
f_{\eta} r f_{\xi} \text { if and only if } \xi \rightarrow^{\prime} \eta,
$$

and $\xi \rightarrow^{\prime}$ core $(S)$ whenever $\xi \in \Gamma \sim\{$ core $(S)\}$. Conversely, if $f_{1}$ is the first element of $F$ then $\left\{x \in E: f_{1}(x)>0\right\}=$ core $(S) \neq \varnothing$, which means that $\Gamma$ has a maximal element with respect to $\rightarrow$. This motivates us to say that a semispace $S$ has a first functional if $S$ has a Klee representation $S(F, r)$ where $F$ has a first member with respect to the linear order $r$, which is equivalent to core $(S)$ being nonempty.

3. Concrete semispaces. It is at this point that we begin our investigation of semispaces in linear topological spaces. For convenience, "locally convex space" will mean "locally convex Hausdorff topological linear space". Subspaces will be assumed to have the relative topology. We denote the topological dual of $E$ by $E^{\prime}$ and continue the use of the notation established in $\$ 2$.

A semispace $S$ in a linear topological space $E$ is called concrete if each $\eta \in \Gamma$ is topologically open in $E_{\eta}$. This means that $\eta$ is a topologically open halfspace in $E_{\eta}$. Our first main result follows.

BASIC REPRESENTATION THEOREM 3.1. A semispace $S$ in a locally convex space $E$ is concrete if and only if $S$ has a Klee representation $S(F, r)$ where $F \subset E^{\prime}$.

Proof. Assume that $S$ is concrete, $\eta \in \Gamma$, and $A$ is the union of all $\xi \rightarrow^{\prime} \eta$. We use the basic separation theorem on the disjoint convex 
sets $\eta$ and $A$ (see 2.2) to obtain a linear functional $g_{\eta}$ on $E_{\eta}$ such that $g_{\eta}(x)>0$ if $x \in \eta$ and $g_{\eta}(x)=0$ if $x \in A$. In fact, $\eta=\left\{x \in E_{\eta}\right.$ : $\left.g_{\eta}(x)>0\right\}$ and by hypothesis $\eta$ is open in $E_{\eta}$ since $S$ is concrete. Therefore $g_{\eta}$ is continuous on $E_{\eta}$. By the Hahn-Banach extension theorem for locally convex spaces there exists for each $\eta$ an extension of $g_{\eta}$ in $E^{\prime}$ and we denote by $f_{\eta}$ one such functional. Let $F=\left\{f_{\eta}\right.$ : $\eta \in \Gamma\}$ and denote by $r$ the linear order on $F$ induced by the reverse of $\rightarrow^{\prime}$ on $\Gamma$. It is shown in Klee's proof of 2.3 that $S=S(F, r)$ and it is easily seen that $S(F, r)$ is a Klee representation for $S$.

Now assume $S$ has a Klee representation $S(F, r)$ where $F \subset E^{\prime}$. Let $\eta \in \Gamma$ and $x \in \eta$ and then note that $\eta=\left\{y \in E: f_{y}=f_{x}\right.$ and $f_{x}(y)>$ $0\}$. If $f_{x}$ is the first member of $F$ then $\eta=\left\{y \in E: f_{x}(y)>0\right\}, E_{\eta}=$ $\eta-\eta=E$, and $\eta$ is open in $E_{\eta}$ since $f_{x} \in E^{\prime}$. Assume then that $f_{x}$ is not the first member of $F$ and define

$$
H=\bigcap_{\substack{z \in \mathcal{E}_{i}(\theta\} \\ x \rightarrow z}} f_{z}^{-1}(0)
$$

The set $H$ is a closed linear subspace of $E$ and we have

$$
\eta=H \cap\left\{y \in E: f_{x}(y)>0\right\}
$$

since $\eta$ consists of all vectors in $E$ which are annihilated by the predecessors of $f_{x}$ and for which $f_{x}$ is positive. Thus $E_{\eta} \subset H$ and $\eta$ is open in $H$ since $F \subset E^{\prime}$ and $\left\{y \in E: f_{x}(y)>0\right\}$ is open in $E$. But the linear span of a nonempty open set is the entire space, so $H=\operatorname{sp}(\eta)=$ $E_{\eta}$. Therefore $\eta$ is open in this and the preceding case which means that $S$ is a concrete semispace and the proof is complete.

Since we did not use the local convexity of $E$ in the second half of the above proof we may conclude that concrete semispaces exist in any linear topological space $E$ whose dual $E^{\prime}$ is total. If $r$ well-orders a total subset $F$ of $E^{\prime} \sim(0)$ then $S(F, r)$ is a concrete semispace in $E$. It is unknown whether or not concrete semispaces exist in linear topological spaces whose duals are not total. If the locally convex space $E$ has finite dimension $n$ and $S$ is a semispace in $E$ with the Klee representation $S(F, r)$, then $F=\left\{f_{1}, \cdots, f_{n}\right\}, F \subset E^{\prime}$, and $S$ is necessarily concrete. It is important to observe that in the infinite-dimensional case a Klee representation for a concrete semispace may utilize discontinuous linear functionals.

We list some elementary properties of semispaces which will be useful later.

LEMma 3.2. Suppose that $S$ is a semispace in a locally convex space $E$.

(a) If the interior of $S$ is not empty then $S$ has a first functional. 


\section{(b) If $S$ has no first functional then $S$ is dense in $E$.}

Proof. (a) This is immediate since interior points are core points. (b) Recall that $-S=S^{*}$ is also a semispace at $\theta$ and $E \sim\{\theta\}=S \cup$ $(-S)$. Suppose $U$ is open and nonempty in $E$. Since neither $S$ nor - $S$ can have interior points according to (a) the cone $S$ must meet $U$ and consequently $S$ is dense in $E$.

LEMMA 3.3. If $S(F, r)$ is any representation for the concrete semispace $S$ in the locally convex space $E$ and $f_{1}$ is the first member of $F$, then $f_{1}$ is continuous and the interior of $S$ is not empty.

We omit the easy proofs of 3.3 and 3.4 .

LEMMA 3.4. A concrete semispace $S$ which is dense in the locally convex space $E$ can have no first functional.

Disjoint subsets $A$ and $B$ of a linear space $E$ are lexicographically separated (see Klee [8]) if there is a semispace $S$ at $\theta$ which contains the algebraic difference set $A-B$. In particular, if $A$ and $B$ are disjoint convex subsets of $E$ then $\theta$ is not in the convex set $A-B$ and a Zorn's lemma argument shows that there is a semispace $S$ at $\theta$ such that $A-B \subset S$. Therefore every pair of disjoint convex subsets of a linear space is separated lexicographically. In a linear topological space we say that $A$ and $B$ are concretely separated (or are separated concretely) provided there is a concrete semispace which separates $A$ and $B$ lexicographically. If $E$ is a finite-dimensional locally convex space, then every disjoint pair of convex sets is concretely separated.

The preceding lemmas permit an easy proof of the following theorem which provides an extremely useful criterion for determining when two sets are not concretely separated.

THEOREM 3.5. If $A$ and $B$ are disjoint subsets of a locally convex space $E$ and if $A-B$ has nonempty core and is dense in $E$, then $A$ and $B$ are not concretely separated.

Proof. If $S$ is any semispace at $\theta$ which contains $A-B$ then $S$ must also have nonempty core and be dense in $E$. Thus $S$ must have a first functional and Lemma 3.4 shows that $S$ cannot be concrete. Therefore $A$ and $B$ cannot be separated concretely.

4. Klee's question concerning lexicographic separation. Following Klee's definition and discussion of lexicographic separation in [8], he asks whether disjoint closed convex subsets of a locally convex 
space $E$ can always be separated lexicographically by a semispace $S(F, r)$ where $F \subset E^{\prime}$. The example which follows satisfies the hypotheses of Theorem 3.5 and Klee's question and consequently it settles the question negatively. This example was motivated by an example of Tukey [12].

ExAmple. There exists a pre-Hilbert space $E$ which contains a disjoint pair of nonempty closed convex subsets $A$ and $B$ such that $A-B$ is dense in $E$ and has nonempty core.

Let $\left(p_{n}\right)=\left(1,2^{-2 / 3}, \cdots, n^{-2 / 3}, \cdots\right)$ in the Hilbert space $l^{2}$. If $G$ denotes the subspace of $l^{2}$ which consists of the finitely nonzero sequences, then let

$$
E=\operatorname{sp}\left(G \cup\left\{\left(p_{n}\right)\right\}\right)=G+\boldsymbol{R}\left(p_{n}\right) .
$$

It follows that

$$
E=\left\{\left(x_{n}\right): \exists n_{0} \in N \text { and } r \in R \ni \text { if } n>n_{0} \text { then } x_{n}=r n^{-2 / 3}\right\}
$$

so that $E_{i}$ consists of those sequences which are eventually $r n^{-2 / 3}$ for some $r \in R$. It was noted by Tukey [12] that

$$
D=\left\{\left(x_{n}\right) \in l^{2}: x_{1} \geqq\left|n\left(x_{n}-n^{-2 / 3}\right)\right|, \text { if } n=2,3, \cdots\right\}
$$

is closed and convex in $l^{2}$. Thus, let $A=D \cap E$ so that $A$ is closed and convex in $E$. If $\left(x_{n}\right) \in A$ and $x_{n}=r n^{-2 / 3}$ for all $n>n_{0}$, then

$$
x_{1} \geqq\left|n\left(r n^{-2 / 3}-n^{-2 / 3}\right)\right|=n^{1 / 3}|r-1|
$$

for all $n>n_{0}$-which is impossible: unless $r=1$. We see that

$$
\begin{aligned}
& A=\left\{\left(x_{n}\right): \exists n_{0} \in N \ni \text { if } 2 \leqq n \leqq n_{0},\right. \text { then } \\
& \left.x_{1} \geqq\left|n\left(x_{n}-n^{-2 / 3}\right)\right| \text { and if } n>n_{0}, \text { then } x_{n}=n^{-2 / 3}\right\} .
\end{aligned}
$$

We define

$$
B=\left\{\left(x_{n}\right) \in E:\left|x_{n}\right| \leqq \frac{1}{2} n^{-2 / 3} \text { for } n=2,3 \cdots\right\}
$$

and note that $B$ is closed and convex in $E$. It is easy to see that $A$ does not meet $B$ and we leave it to the reader to show that $\theta \in$ core $(B)$. Since $\left(p_{n}\right)$ is in $A$ it follows that $\left(p_{n}\right)$ is a core point of $A-B$.

In order to show that $A-B$ is dense in $E$ we let $\varepsilon>0$ be given and suppose that

$$
\left(z_{n}\right)=\left(y_{1}, \cdots, y_{n_{0}}, 0, \cdots\right)+\left(r, \cdots, r n^{-2 / 3}, \cdots\right)
$$

is an arbitrary point in $E$. Since $\left(z_{n}\right)$ and $\left(p_{n}\right)$ are in $l^{2}$ we can find 
positive integers $n_{1}$ and $n_{2}$ such that

$$
\left(\sum_{n=n_{1}}^{\infty}\left(z_{n}\right)^{2}\right)^{1 / 2}<\frac{\varepsilon}{2} \text { and }\left(\sum_{n=n_{2}}^{\infty} n^{-4 / 3}\right)^{1 / 2}<\frac{\varepsilon}{2} .
$$

We let $n_{3}=\max \left\{n_{0}, n_{1}, n_{2}\right\}$ and then define $\left(x_{n}\right) \in A$ by

$$
x_{n}= \begin{cases}\sup _{1 \leqq m \leqq n_{3}}\left\{\left|m\left(z_{m}-m^{-2 / 3}\right)\right|\right\}, & \text { if } n=1 \\ z_{n} & , \text { if } 2 \leqq n \leqq n_{3} \\ n^{-2 / 3} & , \text { if } n>n_{3} .\end{cases}
$$

If $\left(u_{n}\right)$ is defined by

$$
u_{n}= \begin{cases}x_{1}-z_{1}, & \text { if } n=1 \\ 0 & , \text { if } n>1\end{cases}
$$

then $\left(u_{n}\right) \in B$ and, as noted in Tukey [12], $\left(x_{n}\right)-\left(u_{n}\right)$ is within $\varepsilon$ of $\left(z_{n}\right)$. Therefore $A-B$ is dense in $E$.

5. Basic properties of concrete semispaces. We continue our investigation of semispaces in locally convex spaces and then contrast concrete separation with hyperplane separation. The first theorem is a very useful analogue of 2.1.

THEOREM 5.1 Suppose that $E$ is a locally convex space, $M$ is a linear subspace of $E$ and $T$ is a subset of $M$.

(a) $T$ is a concrete semispace in $M$ if there exists a concrete semispace $S$ in $E$ such that $T=S \cap M$.

(b) If $M$ is closed in $E$ and $T$ is a concrete semispace in $M$, then there is a concrete semispace $S$ in $E$ such that $T=S \cap M$.

(c) If $M$ is a closed hyperplane, $Q$ is one of the open halfspaces determined by $M$ and $T$ is a concrete semispace in $M$, then $S=T \cup Q$ is a concrete semispace in $E$ with a first functional.

Proof of (a). According to the basic representation Theorem 3.1 $S$ has a Klee representation $S(F, r)$ with $F \subset E^{\prime}$. By the same theorem, the nonzero restrictions of members of $F$ to $M$ yield a concrete semispace in $M$ when given the order induced by $r$. This semispace is $T$.

Proof of (b). Each vector $x$ in $E \sim M$ admits a linear functional $f$ in $E^{\prime}$ such that $f(x) \neq 0$ and $f(M)=\{0\}$. Let $G_{1}$ be any collection of such functionals which is total for $E \sim M$ and let $r_{1}$ be a wellordering of $G_{1}$. For clarity, assume that any functional in $G_{1}$ which is not a first functional $f_{x}$ for some $x \in E \sim M$ has been deleted. The concrete semispace $T$ has a Klee representation $S\left(G_{2}, r_{2}\right)$ where 
$G_{2} \subset M^{\prime}$, according to 3.1. Extend each member of $G_{2}$ to a linear functional in $E^{\prime}$ and denote this collection by $G$. Now let $F=G_{1} \cup G$ and denote by $r$ the linear order induced on $F$ by $r_{1}$ and $r_{2}$ where it is assumed that $G_{1}$ precedes $G$. Then $S(F, r)$ is a concrete semispace and $T=S(F, r) \cap M$.

Proof of (c). The equivalence classes of $S$ with respect to $\approx$ consist of $Q$ and the equivalence classes of $T$ with respect to $\approx$, so it is easy to see that $S$ is concrete.

CoRollary 5.2. If $A$ and $B$ are subsets of a linear subspace $M$ of the locally convex space $E$ and these subsets are not concretely separated in $M$, then $A$ and $B$ are not concretely separated in $E$.

The proof of 5.2 is based on part (a) of 5.1 .

THEOREM 5.3. Convex subsets of a locally convex space $E$ which are concretely separated by a semispace with first functional are separated by a closed hyperplane.

Proof. Suppose $A$ and $B$ are nonempty convex subsets of $E$ and $S$ is a concrete semispace with first functional such that $A-B \subset S$. If $S(F, r)$ is a Klee representation for $S$ with $F \subset E^{\prime}$ and $f_{1}$ is the first member of $F$, then $A-B \subset\left\{x \in E: f_{1}(x) \geqq 0\right\}$ and $A$ and $B$ are separated by the closed hyperplane $f_{1}^{-1}(\sup f(B))$.

Two subsets $A$ and $B$ of a linear topological space are said to be nicely separated if they are separated by a closed hyperplane $H$ which is disjoint from at least one of the subsets. If $H$ is disjoint from both $A$ and $B$ we say that $A$ and $B$ are strictly separated. The sets are strongly separated provided $H$ lies between two translates of $H$ which separate $A$ and $B$.

THEOREM 5.4. Nicely separated convex subsets $A$ and $B$ of $a$ locally convex space $E$ are concretely separated by a semispace with first functional.

It is clear that the hypothesis of nice separation in 5.4 may be replaced by either strict or strong separation. The following theorem is a consequence of 5.1 and 5.4, but in this case the semispaces are not necessarily at $\theta$.

THeOREM 5.5. Each proper closed convex subset $C$ of a locally convex space $E$ is the intersection of the concrete semispaces with first functionals which contain $C$. 
We now list two simple examples in $\boldsymbol{R}^{2}$ to illustrate some of the differences between concrete and nice separation. Here each disjoint pair of convex sets is concretely separated. The convex sets

$$
X=\{(a, b): b<0\} \cup\{(a, b): a \leqq-1, b=0\}
$$

and

$$
Y=\{(a, b): b>0\} \cup\{(a, b): a \geqq 1, b=0\}
$$

are separated, but are not nicely separated. There is a concrete semispace which contains $X$ and misses $Y$ and vice versa. The closed convex sets

$$
X_{1}=\{(a, b): b \geqq 0\}
$$

and

$$
Y_{1}=\{(a, b): a>0, a b \leqq-1\}
$$

are nicely and concretely separated, but there does not exist a semispace which contains $X_{1}$ and misses $Y_{1}$ nor vice versa.

In this example we exhibit two disjoint bounded convex sets in a normed space which are concretely separated but not separated by a hyperplane (closed or otherwise). Let $E$ be a vector space with a countably infinite Hamel basis $\left\{e_{n}: n \in N\right\}$ and equip $E$ with the sup norm so that $\|x\|=\left\|\Sigma a_{n} e_{n}\right\|=\sup \left\{\left|a_{n}\right|: n \in N\right\}$ (see Dieudonné [2]). There is a corresponding collection of coordinate functionals

$$
F=\left\{f_{n}: n \in N\right\} \text { where } f_{n}(x)=a_{n} \text { if } x=\Sigma a_{n} x_{n} \text {. }
$$

Each $f_{n} \in F$ has norm equal to 1 so $F \subset E^{\prime} \sim\{0\}$.

Assume that $r$ is the linear order for $F$ induced by $>$ from $N$. Form $S=S(F, r)$ and note that $S$ is a concrete semispace without first functional. It is pointed out in Kelley-Namioka [4, page 18] and Köthe [9, page 178] that each vector $x$ in $E$ is the endpoint of a line segment $[a, x]$ such that $[a, x) \subset S$ and $a \neq x$. Hence $S$ and $-S$ cannot be separated by any hyperplane. If $U=\{x \in E:\|x\| \leqq 1\}$ then define

$$
A=S \cap U \text { and } B=-A \text {. }
$$

Both $A$ and $B$ are convex and bounded and $S$ separates them lexicographically. However, any hyperplane which would separate $A$ and $B$ must separate the cones $S$ and $-S$, which is contrary to the above.

6. A characterization of reflexivity for Banach spaces. In this section we direct our attention towards lexicographic separation by 
concrete semispaces in normed linear spaces. If $X$ is a subset of a linear subspace $M$ of a linear space then $\operatorname{core}_{M}(X)$ is the set of core points of $X$ with respect to $M$. We omit the proof of Lemma 6.1.

Lemma 6.1. Suppose $A$ and $B$ are subsets of a linear space and that $s p(A)=L$ and $s p(B)=M$. If $N=L+M, x \in$ core $_{L}(A)$ and $y \in$ core $_{M}(B)$, then $z=x+y$ is in core $_{N}(A+B)$.

The following theorem serves as a further answer to Klee's question discussed in $\$ 4$ and it is the basis for the remaining two major results of this paper. The construction of the sets $A$ and $B$ in the first two paragraphs of the proof is that of Klee as found in the proof of Theorem 4.2 of [6].

THEOREM 6.2. Every nonreflexive separable Banach space $E$ contains a disjoint pair of bounded closed convex sets which cannot be separated concretely.

Proof. Let $F$ be a nonreflexive closed subspace of $E$ of infinite deficiency, which exists according to Theorem 4.1 of [6], and let $C$ be the closed unit ball of $F$. Let $\left\{x_{n}: n \in N\right\}$ be a dense sequence in $\{x \in E:\|x\|=1\}$ and let $K$ be the closed convex hull of

$$
\left\{\theta, x_{1},-x_{1}, \cdots, \frac{1}{n} x_{n},-\frac{1}{n} x_{n}, \cdots\right\} .
$$

This set is compact and $E$ is complete and by a theorem of Mazur [10] it follows that $K$ is compact. If $A$ is the convex hull of $C \cup K$ then $A$ is closed and it can be shown that $\theta \notin$ core $(A)$. It follows that there exists $x_{0}$ in $E \sim\{\theta\}$ so that $(0,1] x_{0} \cap A=\varnothing$. Observe also that $\theta$ is a nonsupport point of $K$ and hence of $A$.

Since $F$ is nonreflexive there is, according to [6, Theorem $(D)]$, a decreasing sequence $C_{1} \supset C_{2} \supset \ldots$ of nonempty bounded closed convex subsets of $F$ such that $\bigcap_{n=1}^{\infty} C_{n}=\varnothing$. We may make further assumptions about the first two sets, namely that $C_{1}=(1 / 2) C$ and $C_{2}=(1 / 4) C$. Note that $t x_{0}+C$ misses $A$ whenever $t>0$. Let $B$ be the closed convex hull of $\bigcup_{n=1}^{\infty}\left((1 / n) x_{0}+C_{n}\right)$. Since the intersection of the sequence $\left\{C_{n}\right\}$ is empty it follows that $B \subset \bigcup_{t>0}\left(t x_{0}+C\right)$ and therefore that $A \cap B=\varnothing$. A proof of the preceding inclusion is found in that of Theorem 1 of Klee [5]. However, the distance from $B$ to $(1 / 2) C$ is zero, so every closed hyperplane separating $A$ from $B$ must contain $C$ and hence support $A$ at $\theta$, which is impossible. Therefore the disjoint bounded closed convex subsets $A$ and $B$ cannot be separated by a closed hyperplane in $E$. 
Now let $N=\operatorname{sp}\left(\left\{x_{0}\right\} \cup A\right)$ and note that $B$ is a subset of $N$. If $L=\operatorname{sp}(A)$ then $L=\bigcup_{n=1}^{\infty} n A$ and $\theta \in \operatorname{core}_{L}(A)$ since $A$ is convex and symmetric about $\theta$. The set $B$ was constructed so that it contains $x_{0}$ and $(1 / 2) x_{0}$. If $M=\boldsymbol{R} x_{0}=\operatorname{sp}(D)$ where $D=\left[(1 / 2) x_{0}, x_{0}\right]$, then $y=$ $(3 / 4) x_{0}$ is core $_{M}(D)$. Since $D \subset B$ and $N=L+M$, it follows from Lemma 6.1 that $-y \in \operatorname{core}_{N}(A-B)$. If $S$ is a semispace at $\theta$ in $N$ which contains $A-B$ then $S$ must have a first functional. If in addition $S$ is a concrete semispace, then Theorem 5.3 implies that $A$ and $B$ are separated in $N$ by a closed hyperplane $H$. The HahnBanach theorem shows that there is a closed hyperplane $H_{1}$ in $E$ such that $H=H_{1} \cap N$. This means that $H_{1}$ separates $A$ from $B$, which is impossible. Therefore $S$ cannot be a concrete semispace in $N$ if $A-B \subset S$. Corollary 5.2 shows that $A$ and $B$ are not concretely separated in $E$ and the proof is complete.

THEOREM 6.3. A Banach space $E$ is reflexive if and only if any two disjoint bounded closed convex subsets of $E$ are concretely separated.

Proof. Tukey [12] proved that when $E$ is reflexive every pair of disjoint bounded closed convex subsets of $E$ is strongly separated. Theorem 5.4 shows that such sets must also be concretely separated.

It is known (see Day [1, page 58], for example) that a Banach space is reflexive if and only if each separable closed subspace is reflexive. The proof is complete as a result of Theorem 6.2.

We conclude with a theorem which yields further insight into the contrast between hyperplane and concrete separation and provides our strongest answer to Klee's question in $\S 4$.

THEOREM 6.4. Every nonreflexive separable Banach space $E$ contains a disjoint pair of bounded closed convex subsets which cannot be separated concretely, but which can be separated by a closed hyperplane.

Proof. There is a closed hyperplane $M$ in $E$ containing $\theta$ which is not reflexive. Construct the disjoint bounded closed convex subsets $A$ and $B$ in $M$ just as was done in the proof of Theorem 6.2. There is a unit vector $x_{1}$ not in $M$ and we define $A_{1}$ to be the convex hull of $\left\{x_{1}\right\} \cup A$ and we define $B_{1}$ to be the convex hull of $\left\{-x_{1}\right\} \cup B$. Observe that $A_{1}$ and $B_{1}$ are disjoint bounded closed convex subsets of $E$ which are nontrivially separated by the closed hyperplane $M$. If $S$ is a concrete semispace which contains $A_{1}-B_{1}$ then part (a) of Theorem 5.1 shows that $S \cap M$ is a concrete semispace in $M$ which contains $A-B$-which is impossible. Therefore $A_{1}$ and $B_{1}$ cannot be concretely separated in $E$. 


\section{REFERENCES}

1. M. M. Day, Normed Linear Spaces, Spring-Verlag, Berlin, 1958.

2. J. Dieudonné, Sur le theoréme de Hahn-Banach, La Revue Scientifique (Paris), 79 (1941), 642-643.

3. P. C. Hammer, Maximal convex sets, Duke Math. J., 22 (1955), 103-106.

4. J. L. Kelley, Namioka and coauthors, Linear Topological Spaces, D. Van Nostrand, Princeton, 1963.

5. V. L. Klee, Some characterizations of reflexivity, Revista de Ciencias (Lima), 52 (1950), 15-23.

6. - Convex sets in linear spaces. II, Duke Math. J., 18 (1951), 877-883.

7. - The structure of semispaces, Math. Scand., 4 (1956), 54-64.

8. - Separation and Support Properties of Convex Sets-a Survey, Control Theory and the Calculus of Variations, Academic Press Inc. New York, (1969), 235-303.

9. G. M. Köthe, Topological Vector Spaces I, Springer-Verlag, New York, 1969.

10. S. Mazur, Uber die kleinste konvexe Menge, die eine gegebene kompakte Menge enhalt, Studia Math., 2 (1930), 7-9.

11. C. E. Moore, Concrete semispaces and a question of Klee, Ph. D. Dissertation, University of Maryland, College Park, Md., 1971.

12. J. W. Tukey, Some notes on the separation of convex sets, Port, Math., 3 (1942), 95-102.

Received September 15, 1971 and in revised form June 22, 1972. This research is contained in the author's doctoral dissertation submitted to the University of Maryland. The author wishes to thank his advisor, Professor George J. Maltese, for the guidance provided during the preparation of that document. This work was supported in part by a United States Naval Academy Research Council grant. A large portion of this material was prepared by the author while at the J. W. Goethe University in Frankfurt am Main, West Germany on Sabbatical Leave from the U. S. Naval Academy.

Department of Mathematics

UNITED States NAVAL ACADEMY

ANNAPOLIS, MARYLAND 21402 


\section{PACIFIC JOURNAL OF MATHEMATICS}

\section{EDITORS}

\section{H. SAMELSON}

Stanford University

Stanford, California 94305

C. R. Новву

University of Washington Seattle, Washington 98105

\section{J. DuGundJI}

Department of Mathematics University of Southern California Los Angeles, California 90007

RICHARD ARENS

University of California Los Angeles, California 90024

\section{ASSOCIATE EDITORS}
E. F. BECKENBACH
B. H. NeumanN
F. WOLF
K. YoSHIDA

\section{SUPPORTING INSTITUTIONS}

\author{
UNIVERSITY OF BRITISH COLUMBIA \\ CALIFORNIA INSTITUTE OF TECHNOLOGY \\ UNIVERSITY OF CALIFORNIA \\ MONTANA STATE UNIVERSITY \\ UNIVERSITY OF NEVADA \\ NEW MEXICO STATE UNIVERSITY \\ OREGON STATE UNIVERSITY \\ UNIVERSITY OF OREGON \\ OSAKA UNIVERSITY
}

\author{
UNIVERSITY OF SOUTHERN CALIFORNIA \\ STANFORD UNIVERSITY \\ UNIVERSITY OF TOKYO \\ UNIVERSITY OF UTAH \\ WASHINGTON STATE UNIVERSITY \\ UNIVERSITY OF WASHINGTON \\ $*{ }^{*}$
AMERICAN MATHEMATICAL SOCIETY
NAVAL WEAPONS CENTER
}

The Supporting Institutions listed above contribute to the cost of publication of this Journal, but they are not owners or publishers and have no responsibility for its content or policies.

Mathematical papers intended for publication in the Pacific Journal of Mathematics should be in typed form or offset-reproduced, (not dittoed), double spaced with large margins. Underline Greek letters in red, German in green, and script in blue. The first paragraph or two must be capable of being used separately as a synopsis of the entire paper. The editorial "we" must not be used in the synopsis, and items of the bibliography should not be cited there unless absolutely necessary, in which case they must be identified by author and Journal, rather than by item number. Manuscripts, in duplicate if possible, may be sent to any one of the four editors. Please classify according to the scheme of Math. Rev. Index to Vol, 39. All other communications to the editors should be addressed to the managing editor, Richard Arens, University of California, Los Angeles, California, 90024.

50 reprints are provided free for each article; additional copies may be obtained at cost in multiples of 50 .

The Pacific Journal of Mathematics is issued monthly as of January 1966. Regular subscription rate: $\$ 48.00$ a year (6 Vols., 12 issues). Special rate: $\$ 24.00$ a year to individual members of supporting institutions.

Subscriptions, orders for back numbers, and changes of address should be sent to Pacific Journal of Mathematics, 103 Highland Boulevard, Berkeley, California, 94708.

PUBLISHED BY PACIFIC JOURNAL OF MATHEMATICS, A NON-PROFIT CORPORATION

Printed at Kokusai Bunken Insatsusha (International Academic Printing Co., Ltd.), 270, 3-chome Totsuka-cho, Shinjuku-ku, Tokyo 160, Japan. 


\section{Pacific Journal of Mathematics}

\section{Vol. 44, No. $2 \quad$ June, 1973}

Tsuyoshi Andô, Closed range theorems for convex sets and linear liftings . . . . . . 393

Richard David Bourgin, Conically bounded sets in Banach spaces . . . . . . . . . 411

Robert Jay Buck, Hausdorff dimensions for compact sets in $R^{n} \ldots \ldots \ldots \ldots \ldots \ldots . \ldots 421$

Henry Cheng, A constructive Riemann mapping theorem ................ 435

David Fleming Dawson, Summability of subsequences and stretchings of

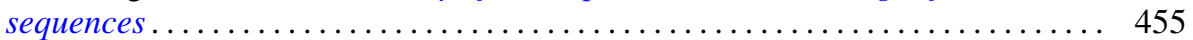

William Thomas Eaton, A two sided approximation theorem for 2-spheres ....... 461

Jay Paul Fillmore and John Herman Scheuneman, Fundamental groups of compact complete locally affine complex surfaces ....................... 487

Avner Friedman, Bounded entire solutions of elliptic equations . . . . . . . . . . . 497

Ronald Francis Gariepy, Multiplicity and the area of an $(n-1)$ continuous

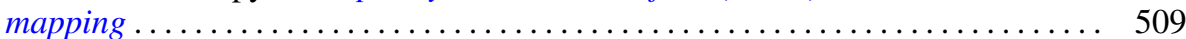

Andrew M. W. Glass, Archimedean extensions of directed interpolation groups . . . . 515

Morisuke Hasumi, Extreme points and unicity of extremum problems in $H^{1}$ on

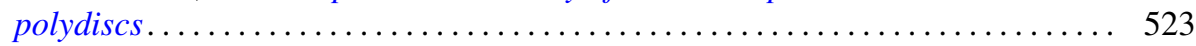

Trevor Ongley Hawkes, On the Fitting length of a soluble linear group . . . . . . 537

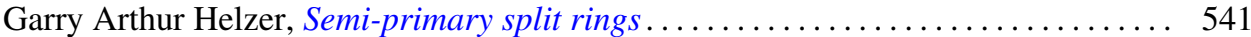

Melvin Hochster, Expanded radical ideals and semiregular ideals . . . . . . . . . 553

Keizō Kikuchi, Starlike and convex mappings in several complex variables . . . . . . 569

Charles Philip Lanski, On the relationship of a ring and the subring generated by its

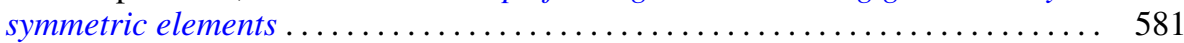

Jimmie Don Lawson, Intrinsic topologies in topological lattices and semilattices ........................................... 593

Roy Bruce Levow, Counterexamples to conjectures of Ryser and de Oliveira ...... 603

Arthur Larry Lieberman, Some representations of the automorphism group of an infinite continuous homogeneous measure algebra ..........

William George McArthur, $G_{\delta}$-diagonals and metrization theorems $\ldots .$.

James Murdoch McPherson, Wild arcs in three-space. II. An invariant of

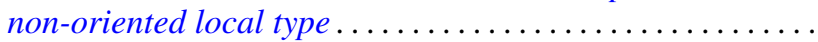

H. Millington and Maurice Sion, Inverse systems of group-valued measures ...

C. Edward Moore, Concrete semispaces and lexicographic separation of convex

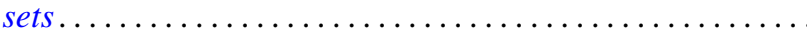

Jingyal Pak, Actions of torus $T^{n}$ on $(n+1)$-manifolds $M^{n+1}$.

Merrell Lee Patrick, Extensions of inequalities of the Laguerre and Turán type . . . . 675

Harold L. Peterson, Jr., Discontinuous characters and subgroups of finite index. . . . 683

S. P. Philipp, Abel summability of conjugate integrals . . . . . . . . . . . . . 693

R. B. Quintana and Charles R. B. Wright, On groups of exponent four satisfying an

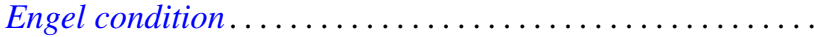

Marlon C. Rayburn, On Hausdorff compactifications. . . . . . . . . .

Martin G. Ribe, Necessary convexity conditions for the Hahn-Banach theorem in

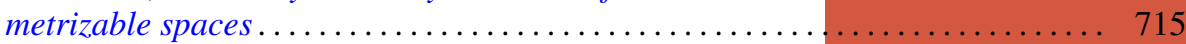

Ryōtarō Satō, On decomposition of transformations in infinite measure spaces .... 733

Peter Drummond Taylor, Subgradients of a convex function obtained from a

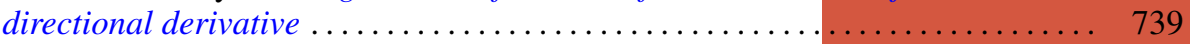

James William Thomas, A bifurcation theorem for $k$-set contractions . . . . . . . . 749 Clifford Edward Weil, A topological lemma and applications to real functions . . . . 757

Stephen Andrew Williams, A nonlinear elliptic boundary value problem . . ....... 767

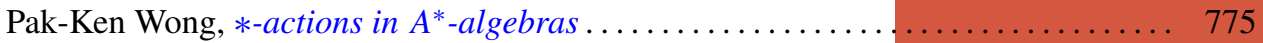

